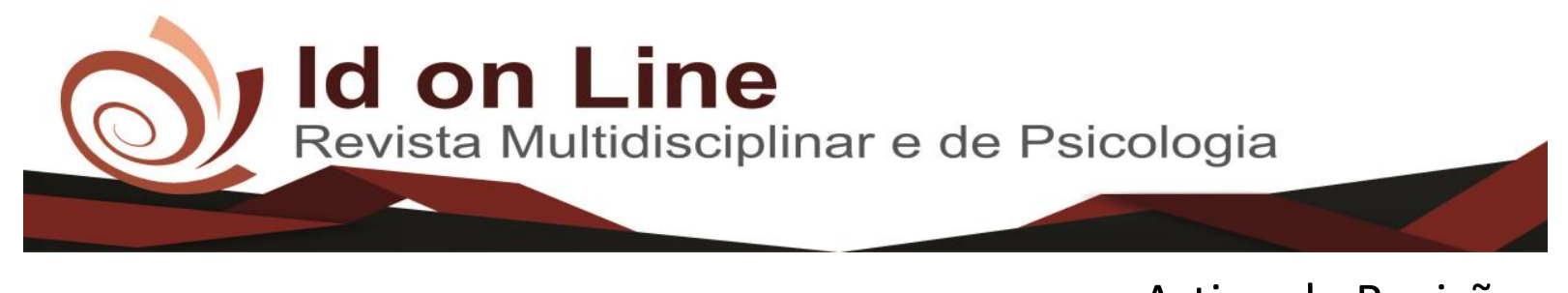

Artigo de Revisão

\title{
Aspectos relacionados à ocorrência de Flare-Up em Endodontia: Revisão da Literatura
}

\author{
Laurentino Alves Brito Neto ${ }^{1}$, Marcelo Pereira da Rocha ${ }^{2}$
}

\begin{abstract}
Introdução: Flare-up endodôntico é uma situação desagradável para o paciente e cirurgião-dentista e pode influenciar de maneira direta na relação destes. Objetivo: revisar a literatura acerca dos principais aspectos relacionados com a ocorrência de flare-ups em endodontia. Metodologia: trata-se revisão da literatura, utilizandose acesso às bases de dados SciELO, PUBMED e LILACS, com estudos publicados no período 2014 a 2019. Resultados: os estudos apontaram baixa incidência de flare-up. Os fatores relacionados à sua ocorrência foram estados pulpar, dor prévia ao tratamento, idade e sexo. Por sua vez, técnica de instrumentação, solução irrigadora, posição do dente, número de canais, ampliação foraminal, medicação intracanal e número de sessões clínicas não tiveram relação significativa com a sua ocorrência. Conclusão: Medidas de prevenção, amplamente conhecidas, devem ser adotadas durante a intervenção endodôntica, pois reduzem a ocorrência de flare-up.
\end{abstract}

Palavras-Chave: Endodontia. Canal Radicular. Complicações Endodônticas.

\section{Aspects related to the occurrence of Flare-Up in Endodontics: Literature Review}

\begin{abstract}
Introduction: Endodontic Flare-up is an unpleasant situation for the patient and dentist and can directly influence their relationship. Objective: To review the literature on the main aspects related to the occurrence of flare-ups in Endodontics. Methodology: This is a review of the literature, using access to the databases SciELO, PUBMED and LILACS, with studies published in the period 2014 to 2019. Results: The studies showed a low incidence of flare-up. The factors related to its occurrence were pulp, pain prior to treatment, age and sex. In turn, instrumentation technique, irrigating solution, tooth position, number of canals, foraminal enlargement, intracellular medication and number of clinical sessions were not significantly related to its occurrence. Conclusion: Preventive measures, widely known, should be adopted during endodontic intervention, as they reduce the occurrence of flare-up.
\end{abstract}

Keywords: Endodontics. Root Canal. Endodontic complications.

\section{Introdução}

A dor entre consultas ou após a conclusão do tratamento endodôntico é uma situação desagradável para o paciente e cirurgião-dentista e pode influenciar de maneira direta na relação destes (FRANCISCO et al., 2016). Sendo assim, o profissional deve

\footnotetext{
${ }^{1}$ Discente do Curso de Odontologia da Faculdade Independente do Nordeste (FAINOR). labneto67@ outlook.com;

${ }^{2}$ Docente do Curso de Odontologia da Faculdade Independente do Nordeste (FAINOR), Mestre em Saúde Coletiva. cdbiomarcelo@yahoo.com.br.
} 
empregar medidas adequadas a fim de impedir o desenvolvimento de episódios dolorosos associados aos tratamentos endodônticos (BIDAR et al., 2015).

O sucesso da terapia endodôntica pode ser descrito como a manutenção do dente na cavidade bucal em função, sem prejuízos à saúde do paciente. Para tanto, o tratamento endodôntico deve seguir princípios científicos e biológicos para que sejam reduzidas as possibilidades de falhas e acidentes (TOIA, 2018). O fator microbiológico tem recebido destaque na literatura especializada, pois a maioria das doenças pulpares e dos tecidos periapicais está direta ou indiretamente relacionada ao desenvolvimento de microrganismos (GABARDO et al., 2009).

Ressalta-se o avanço significativo do ponto de vista tecnológico com consequente transformação na Endodontia, a exemplo do desenvolvimento de novas técnicas, introdução de novos equipamentos e materiais. Com a presença de tecnologias modernas houve redução do tempo operatório do tratamento, gerando maior conforto para o paciente e menor estresse para o profissional (SANTOS et al., 2018).

Apesar do elevado nível de sucesso da terapia endodôntica e de ter se demonstrado um procedimento previsível, fracassos ocorrerem após o tratamento, seja por persistência da infecção ou por recontaminação dos canais em algum momento após a intervenção. A taxa de insucesso pode variar, a depender da condição pulpar, e no geral é de 15\% (WALTON; FOUAD, 1992; YAYLALI et al., 2018).

Mesmo com os avanços na odontologia, a sintomatologia dolorosa ainda é uma realidade no pós-operatório endodôntico. Flare-up é definido como uma exacerbação aguda da condição pulpar ou perirradicular após início do tratamento endodôntico (SAOUD et al., 2016). É uma complicação alarmante para o paciente e desafiadora para o cirurgião-dentista. A prevenção de sua ocorrência é, portanto, muito desejada (EHRMANN; MESSER; CLARK, 2007). A dor associada ao flare-ups pode ser considerada como extrema e insuportável que pode repercutir para complicações físicas e emocionais (BIDAR et al., 2015; SIPAVIČIŪTĖ; MANELIENĖ, 2014).

Frente ao exposto, este estudo objetivou revisar a literatura acerca dos principais aspectos relacionados com a ocorrência de flare-ups em endodontia. 


\section{Metodologia}

Trata-se de revisão de literatura realizada com acesso à internet, buscando-se uma síntese do conhecimento da temática em questão, partindo da seguinte pergunta de pesquisa: quais são os principais fatores de risco e incidência dos flare-ups em Endodontia? Para tanto, foram utilizadas as seguintes palavras-chave para a busca dos estudos pesquisados: endodontia e flare-up. O acesso dos trabalhados foi realizado por meio das bases de dados: Scientific Electronic Library (SciELO), PUBMED, Literatura Latino-Americana e do Caribe em Ciências da Saúde (LILACS).

O período pesquisado englobou os anos de 2014 a 2019, com intuito de obter acesso aos trabalhos mais recentes sobre o assunto em questão. Foram incluídos somente artigos científicos, dentro da temática proposta, selecionado por meio da leitura do título e do resumo, com textos completos, publicados nas línguas portuguesa e inglesa. Por sua vez, foram adotados os seguintes critérios de exclusão: trabalhos em outro formato que não artigo, como cartas ao editor, editoriais, resumos, teses e dissertações; os que fogem da temática proposta, artigos incompletos e publicados em outros idiomas que não os citados anteriormente.

Os artigos foram catalogados em planilha e analisados a fim de se identificar duplicatas, após seleção com base nos títulos e resumos lidos. Uma análise descritiva foi realizada de modo a fornecer uma síntese do conhecimento mais atualizado sobre o assunto.

\section{Ocorrência de Flare-Up em Endodontia}

A ocorrência de flare-up após uma consulta de tratamento do canal radicular é um problema significativo. Isso é perturbador tanto para o paciente quanto para o dentista e atrapalha a prática clínica (SIPAVIČIŪTĖ; MANELIENĖ, 2014). O fenômeno é complexo e, embora pouco compreendido, envolve, sem dúvida, vários aspectos relacionados às alterações teciduais locais, fatores microbianos, fenômenos imunológicos e outras entidades (M SEDIGH et al., 2012).

Flare-ups são situações indesejáveis, pois requerem uma visita não programada e causa grande desconforto ao paciente devido à dor e edema. Embora as razões exatas para sua ocorrência não sejam claramente compreendidas, é uma condição definitivamente de natureza multifatorial (NAIR et al., 2017). 
Um estudo discutiu aspectos relacionados a frequência e fatores associados à flare-ups em tratamentos endodônticos. Para os autores, permanecem determinadas controvérsias em relação a fatores, como sexo, idade, posição do dente e diagnostico pulpar, técnica de instrumentação, soluções irrigadoras e medicação intracanal, bem como número de sessões clínicas. Concluíram que presença de comprometimento periapical representou um forte preditor a ocorrência de flare-ups (FONTENELE et al., 2016).

Pesquisa registrou $2 \%$ de incidência de flare-up associado à terapia endodôntica. A idade do paciente, sexo e diagnóstico tiveram um efeito significativo no seu desenvolvimento $(\mathrm{p}<0,05)$. O tipo e posição do dente, número de canais radiculares, número de visitas e modalidade de tratamento não tiveram efeito significativo na incidência. $O$ diagnóstico desempenha um papel importante na prevenção da incidência. Pacientes na faixa etária de 40 a 60 anos apresentaram maior risco de desenvolver flare-up. As mulheres, em comparação com os homens, são mais propensas (NAIR et al., 2017).

Um total de 106 dentes tratados endodonticamente foram analisados em pacientes com

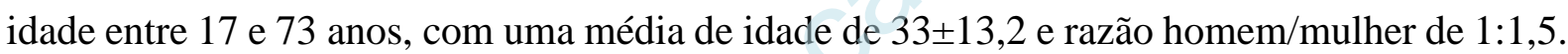
A incidência de flare-up foi de $8,5 \%$. Antes do tratamento, $47 \%$ dos casos tinham dor, $61,3 \%$ tinham radiolucidez apical e $83 \%$ com necrose pulpar. A maioria $(7,77,8 \%)$ do flare-up ocorreu após a primeira visita. Apenas a dor prévia ao tratamento teve uma relação estatisticamente significativa com a sua ocorrência $(\mathrm{p}=0,009)$ (ALVES, 2010; ONAY; UNGOR; CANAN YAZICI, 2015; WALTON; FOUAD, 1992).

Outro estudo avaliou 1.500 registros de pacientes em prontuários revisados, e encontrou uma prevalência de 2,3\% de flare-up. O estado do espaço do canal radicular dos pacientes (vital, não vital ou retratamento), condição médica e demografia (idade, sexo, tipo de dente e posição) foram observados. Novecentos e cinquenta e um registros de pacientes preencheram os critérios de inclusão. Houve uma correlação entre o estado do espaço do canal e a idade do paciente com o desenvolvimento do agravo $(\mathrm{p}<0,05)$. Não houve associação entre ocorrência de flare-up e tipo de dente, localização, sexo ou condição médica (p>0,5) (AZIM; AZIM; ABBOTT, 2017).

Pesquisa comparou a incidência e intensidade da dor pós-operatória e flare-ups entre pulpectomia em visita única e múltipla sessão em molares decíduos. Além disso, correlacionou o estado pré-operatório da polpa à dor pós-operatória. Os dentes do grupo um (pulpectomia em visita única) foram obturados na mesma visita. Os dentes do grupo dois (pulpectomia em múltiplas visitas) foram obturados na consulta subsequente. O registro de dor pós-operatória, flare-up e uso de medicação foi feito após 24 horas, sete dias e um mês. Quatro casos em ambos 
os grupos relataram dor pós-operatória (10\%) na análise de 24 horas, p=0,74. Um caso de flareup $(2,5 \%)$ foi registrado em cada grupo $\mathrm{p}=0,67$. Nenhum dos pacientes relatou dor no sétimo dia e um mês após atendimento. A dor pós-operatória foi registrada em cinco dentes não vitais $(13,5 \%)$ e três dentes vitais $(6,9 \%)$. Houve baixa incidência de dor pós-operatória. A maioria dos pacientes em ambos os grupos relatou ausência de dor ou dor mínima nas 24 horas de tratamento. Não houve diferenças entre os protocolos de tratamento de visita única e múltipla em relação à incidência de dor pós-operatória. Nenhuma correlação significativa foi encontrada entre a vitalidade pulpar e a incidência de dor pós-operatória (SEVEKAR; GOWDA, 2017).

Por meio de revisão da literatura buscaram-se verificar se a conclusão do tratamento do canal radicular em uma única visita ou mais de duas ou mais visitas, com ou sem medicação faz alguma diferença em termos de eficácia ou complicações. O estudo concluiu que não há evidências que um regime de tratamento de canal radicular em sessão única ou em duas ou mais sessões seja melhor que o outro. Nenhum deles pode prevenir todas as complicações a curto e longo prazo (MANFREDI et al., 2016).

Outro estudo comparou o efeito de dois diferentes instrumentos rotatórios na dor pósoperatória em dentes com pulpite irreversível assintomática. Um total de 78 primeiros e segundos molares inferiores foram divididos em dois grupos $(n=39)$ e o preparo do canal radicular foi realizado com instrumentos rotatórios $\mathrm{RaCe}$ ou ProTaper. Todos os indivíduos foram submetidos a tratamento de canal único em uma visita e a severidade da dor pósoperatória foi avaliada por meio da escala visual analógica em intervalos de 4, 12, 24, 48 e 72 horas e uma semana. Além disso, a necessidade de tomar analgésicos foi registrada. A comparação da média da intensidade da dor entre os dois grupos em vários intervalos pósoperatórios não revelou diferenças significativas $(\mathrm{p}=0,10)$. A diferença na quantidade de analgésicos tomados por cada grupo não foi estatisticamente significativa $(p=0,25)$. Não houve diferenças significativas na dor pós-operatória relatada entre os dois grupos, o que indica a aceitabilidade clínica de ambos os sistemas (SHAHI et al., 2016).

Objetivando avaliar a dor pós-operatória, foi realizado ensaio clínico prospectivo randomizado comparando dois grupos, utilizando o sistema Reciproc® em um grupo e o sistema rotatório ProTaper® no outro. O estudo incluiu 78 pacientes do sexo masculino, com idade entre 18 e 64 anos (idade média de 26 anos), com necrose pulpar assintomática em dentes molares inferiores $(n=78)$. O tratamento endodôntico em sessão única foi realizado por um único operador especializado em Endodontia. A preparação mecânica dos canais radiculares foi realizada utilizando as técnicas de instrumentação ProTaper® e Reciproc®. A dor pós- 
operatória foi registrada por meio de uma escala verbal de classificação e descrição verbal com categorias bem definidas nos três intervalos de tempo seguintes: 24 horas, 72 horas e 7 dias após o procedimento endodôntico. A avaliação da dor pós-operatória foi registrada como ausência de dor, dor leve, dor moderada e dor ou surto intenso. A incidência de dor pósoperatória no grupo ProTaper (PT) 24 h após o procedimento endodôntico foi de 17,9 e 5,1\% após 72 h. No grupo Reciproc (RP), a incidência após 24 h foi de 15,3 e 2,5\% após 72 h. Nenhum paciente apresentou dor intensa nos intervalos de tempo avaliados. Não houve diferença significativa $(p>0,05)$ na dor pós-operatória entre a técnica de instrumentação ProTaper® e Reciproc ${ }^{\circledR}$ durante o tratamento endodôntico (RELVAS et al., 2016).

Quatro grupos divididos de acordo com a idade dos pacientes foram avaliados. Dos 50 pacientes na faixa etária de 21 a 30 anos, apenas quatro apresentaram exacerbação do quadro endodônticos pós-tratamento, enquanto nenhum caso foi registrado em pacientes com faixa etária de 31 a 50 anos. Apenas dois homens e quatro mulheres apresentaram crises no pósoperatório. Uma correlação não significativa foi obtida quando os casos de dor foram comparados com base no tipo de solução de irrigação usada durante a preparação do canal (PRIYANK et al., 2016).

Outra pesquisa teve como objetivo avaliar a eficácia de duas doses orais únicas de betametasona previamente ao tratamento sobre a incidência de exacerbação entre consultas e desconforto pós-operatório. Cinquenta e quatro pacientes com idade entre 18 e 59 anos que necessitaram de tratamento endodôntico foram selecionados e distribuídos aleatoriamente em três grupos; dose única pré tratamento oral de placebo ou betametasona em duas doses orais diferentes de $0,5 \mathrm{mg}$ e $1 \mathrm{mg}$, respectivamente. A terapia endodôntica foi completada em duas visitas usando pasta antibiótica tripla como medicamento intracanal. Os pacientes receberam um questionário para registrar sua dor em 1, 2, 3 e 7 dias após o tratamento. Na segunda visita, a obturação foi feita, e os pacientes foram novamente instruídos a registrar seus escores de dor após o tratamento e alta. $\mathrm{O}$ grupo de $0,5 \mathrm{mg}$ de betametasona apresentou menores escores médios de dor entre todos os grupos experimentais; no entanto, não houve diferença estatisticamente significativa entre nenhum dos grupos ( $p>0,05)$. A dose oral única de betametasona no pré-tratamento é eficaz na prevenção de flare-ups endodônticos; no entanto, os resultados foram estatisticamente insignificantes. (GYANANI; CHHABRA; PARMAR, 2016).

Com o intuito de compreender a relação entre o aumento foraminal e a dor pósoperatória em endodontia foi realizada revisão sistemática da literatura com metanálise, com 
inclusão de cinco estudos. O estudo demonstrou que o aumento foraminal proporciona maior dor nos primeiros dias. A dor foi maior no tratamento endodôntico com alargamento do forame, com diferença estatisticamente significativa no primeiro, segundo quarto, sexto e sétimo dia. Dados limitados indicaram que não houve diferença significativa no consumo de analgésicos, flare up e edema. A dor pós-operatória foi maior no grupo com alargamento foraminal em comparação com a terapia endodôntica convencional nos primeiros dias após o tratamento em dentes com necrose e periodontite apical (BORGES SILVA et al., 2017).

Relato clínico apresentado por Gonçalves et al., (2016) mostrou que um paciente de 47 anos relatou dor na percussão após retratamento sem complicações do canino maxilar esquerdo para fins protéticos. Nos dias seguintes, a dor aumentou dramaticamente, levando à remoção da obturação e uso de medicação intracanal. Depois de muitas tentativas frustradas de resolver o problema, uma cultura microbiana do canal radicular detectou a presença de Staphylococcus epidermidis. Um antibiograma determinou a melhor combinação de drogas para controlar esta infecção: tetraciclina (cloridrato de oxitetraciclina, $500 \mathrm{mg}$ por via oral) e cefalosporina de terceira geração (ceftriaxona, um grama por via intramuscular). Uma vez controlada a infecção, o canal radicular foi obturado. Houve redução na área de radiolucidez e o paciente não relatou dor após dois anos de acompanhamento.

\section{Discussão}

Os atendimentos de urgências, como flare-up, são apresentados como condições nas quais o paciente procura a assistência por sentir dor aguda ou edema, o que requer atendimento imediato para alívio dos sintomas (AZIM; AZIM; ABBOTT, 2017). É um quadro muito angustiante para o paciente e para o operador (SEVEKAR; GOWDA, 2017).

O problema é grave e repercute de forma negativa (AZIM; AZIM; ABBOTT, 2017; NAIR et al., 2017). Demanda visita não programada e tratamento ativo (incisão para drenagem, abertura endodôntica, dentre outros procedimentos). Devido à sua natureza de urgência, criamse dificuldades tanto para o paciente quanto para o dentista (JR, 2003; NAIR et al., 2017; SEVEKAR; GOWDA, 2017).

Destacam-se metodologias diferenciadas entre os estudos encontrados o que dificulta a comparação entre os mesmos e não há consenso acerca de alguns fatores predisponentes. Sabese que o flare-up está relacionado a infecção bacteriana e a extrusão apical de detritos durante 
a intervenção endodôntica. Desta forma, acrescentam que culturas microbianas e antibiogramas devam ser usados para permitir o manejo mais rápido e bem-sucedido de lesões refratárias.

Fontenele et al., (2016) destacam que alguns fatores como sexo, idade, técnica de instrumentação, solução irrigadora, dentre outros, não tem correlação com o flare-up bem evidenciada e destacam que o comprometimento periapical é um forte preditor. A dor prévia ao tratamento tem relação significativa com a sua ocorrência. Por sua vez, outro estudo mostrou relação do flare-up com a idade e sexo, sendo que pacientes de 40 a 60 anos são mais propensos e atinge em maior escala as mulheres (NAIR et al., 2017). O trabalho de Azim, Azim e Abbott (2017) demonstrou correlação entre o estado pulpar e idade do paciente com a ocorrência dessa urgência endodôntica.

Nair et al., (2017) encontraram incidência de flare-up em 2\% das terapias endodônticas. Pesquisa realizada por Azim, Azim e Abbott (2017), cuja incidência, foi de 2,3\%, parecem coadunar com a baixa ocorrência desse quadro.

No entanto, o número de sessões para realização da terapia endodôntica não interfere na ocorrência de flare-ups Com base nas evidências disponíveis é possível afirmar os benefícios do tratamento em sessão única em termos de tempo e conveniência, tanto para o paciente quanto para o dentista, apesar de maio frequência de dor pós-operatória e consequente uso de analgésicos (FONTENELE et al., 2016; NAIR et al., 2017)

Quando se analisaram solução irrigadora utilizada durante os tratamentos de canais radiculares com a incidência de flare-up, os estudos não apontaram relação significativa (PRYANK et al., 2016; FONTENELE et al., 2016)

De acordo com resultados encontrados, técnicas rotatórias e reciprocantes bem conhecidas e utilizadas mundialmente não interferiram na ocorrência de flare-up. A incidência de dor pós-operatória foi baixa e semelhante entre os estudos realizados (RELVAS et al., 2016)

A dose oral única de betametasona no pré-tratamento revelou-se eficaz no tratamento de flare-ups endodônticos; no entanto, os resultados foram estatisticamente insignificantes. (GYANANI; CHHABRA; PARMAR, 2016). Por outro lado, os autores aconselharam uma abordagem cautelosa e racional quando se considera o aumento foraminal durante endodontia, apesar de não ficar comprovada a utilização dessa técnica com a ocorrência de flare-up (BORGES SILVA et al., 2017).

Um único estudo encontrado (SEVERKAR; GAUDE., 2017) pesquisou a ocorrência de flare-up em dentes decíduos e demonstrou baixa ocorrência desse envento. Evidência grande 
lacuna de conhecimento neste ponto, apesar de já haver muita pesquisa relacionando a sua ocorrência com a idade adulta (AZIM; AZIM; ABBOTT, 2017).

\section{Conclusões}

Os estudos encontrados apontam para uma baixa incidência de flare-up associado ao tratamento endodôntico, apesar das ressalvas quanto às diferenças metodológicas entre os trabalhos, fato que demanda novas pesquisas sobre o assunto.

Quanto aos fatores relacionados à incidência de flare-up foram encontradas correlações com estado pulpar, dor prévia ao tratamento, idade e sexo. Por sua vez, fatores como técnica de instrumentação, solução irrigadora, posição do dente, número de canais, ampliação foraminal, medicação intracanal e número de sessões clínicas não tiveram relação significativa com a sua ocorrência.

Fica bastante evidente os transtornos para o paciente e profissional assistente, tendo-se em vista a gravidade do quadro clínico, o que requer consulta de urgência e pode comprometer a relação profissional/paciente. Desta forma, as medidas de prevenção, amplamente conhecidas, devem ser adotadas durante a intervenção endodôntica, a fim de se reduzir a ocorrência desse agravo.

\section{Referências}

ALVES, V. D. O. Endodontic flare-ups: A prospective study. Oral Surgery, Oral Medicine, Oral Pathology, Oral Radiology and Endodontology, v. 110, n. 5, p. e68-e72, 2010.

AZIM, A. A.; AZIM, K. A.; ABBOTT, P. V. Prevalence of inter-appointment endodontic flareups and host-related factors. Clinical Oral Investigations, v. 21, n. 3, p. 889-894, 2017.

BIDAR, M. et al. A survey over the dentists??? and endodntists??? approaches towards the management of endodontic emergencies in Mashhad, Iran. Iranian Endodontic Journal, v. 10, n. 4, p. 256-262, 2015.

BORGES SILVA, E. A. et al. Evaluation of Effect of Foraminal Enlargement of Necrotic Teeth on Postoperative Symptoms: A Systematic Review and Meta-analysis. Journal of Endodontics, v. 43, n. 12, p. 1969-1977, 2017.

EHRMANN, E. H.; MESSER, H. H.; CLARK, R. M. Flare-ups in endodontics and their relationship to various medicaments. p. 119-130, 2007. 
FONTENELE, J. W. N. et al. INCIDÊNCIA E FATORES ASSOCIADOS A FLARE-UPS EM ENDODONTIA: REVISÃO DE LITERATURA. Salusvita, v. 35, n. 4, p. 547-561, 2016.

FRANCISCO, S. S. et al. Clinical strategies for managing emergency endodontic pain. Revista Sul-Brasileira de Odontologia, v. 13, n. 3, p. 209-216, 2016.

GABARDO, M. C. L. et al. Microbiologia do insucesso do tratamento endodôntico. Revista Gestão \& Saúde, v. 01, n. 01, p. 11-17, 2009.

GYANANI, H.; CHHABRA, N.; PARMAR, G. Comparative assessment of efficacy of two different pretreatment single oral doses of betamethasone on inter-appointment and postoperative discomfort: An in vivo clinical evaluation. Journal of Conservative Dentistry, v. 19, n. 6, p. 564, 2016.

JR, J. F. S. Micro1. International Endodontic Journal, p. 1-11, 2003.

M SEDIGH, S. et al. Comparison of Flare up Incidence in Patients Treated by Different Practitioners. Journal of Dentistry (17283426), v. 13, n. 4, p. 164-168, 2012.

MANFREDI, M. et al. Single versus multiple visits for endodontic treatment of permanent teeth. Cochrane Database of Systematic Reviews, n. 12, 1 dez. 2016.

NAIR, M. et al. Incidence of Endodontic Flare-ups and Its Related Factors: A Retrospective Study. Journal of International Society of Preventive and Community Dentistry, v. 7, p. 175-9, 2017.

ONAY, E. O.; UNGOR, M.; CANAN YAZICI, A. The evaluation of endodontic flare-ups and their relationship to various risk factors. BMC Oral Health, v. 15, n. 1, p. 1-5, 2015.

PRIYANK, H. et al. Assessment of the incidence of posttreatment endodontic flare-ups in patients undergoing single-sitting root canal therapies: A clinical study. Journal of Contemporary Dental Practice, v. 17, n. 10, p. 849-852, 2016.

RELVAS, J. B. F. et al. Assessment of postoperative pain after reciprocating or rotary NiTi instrumentation of root canals: a randomized, controlled clinical trial. Clinical Oral Investigations, v. 20, n. 8, p. 1987-1993, 2016.

SANTOS, F. L. P. et al. Conhecimento dos Acadêmicos de Odontologia Sobre a Dor PósOperatória em Endodontia. Revista de Iniciação Científica em Odontologia, v. 16, n. 3, p. 515, 2018.

SAOUD, T. et al. Regeneration and Repair in Endodontics-A Special Issue of the Regenerative Endodontics-A New Era in Clinical Endodontics. Dentistry Journal, v. 4, n. 1, p. 3, 2016.

SEVEKAR, S. A.; GOWDA, S. H. N. Postoperative pain and flare-ups: Comparison of incidence between single and multiple visit pulpectomy in primary molars. Journal of Clinical and Diagnostic Research, v. 11, n. 3, p. ZC09-ZC12, 2017. 
SHAHI, S. et al. Postoperative pain after endodontic treatment of asymptomatic teeth using rotary instruments: A randomized clinical trial. Iranian Endodontic Journal, v. 11, n. 1, p. 38-43, 2016.

SIPAVIČIŪTĖ, E.; MANELIENĖ, R. Pain and flare-up after endodontic treatment procedures. Stomatologija, v. 16, n. 1, p. 25-30, 2014.

TOIA, C. Tratamento Endodôntico em Sessão Única X Múltiplas Sessões: correlação do sucesso após 1 ano de tratamento com níveis de endotoxinas, carga microbiana e sinais/sintomas. p. 41, 2018.

WALTON, R.; FOUAD, A. Endodontic interappointment flare-Ups: A prospective study of incidence and related factors. Journal of Endodontics, v. 18, n. 4, p. 172-177, 1992.

YAYLALI, I. E. et al. Does Maintaining Apical Patency during Instrumentation Increase Postoperative Pain or Flare-up Rate after Nonsurgical Root Canal Treatment? A Systematic Review of Randomized Controlled Trials. Journal of Endodontics, v. 44, n. 8, p. 1228-1236, 2018.

\section{Como citar este artigo (Formato ABNT):}

BRITO NETO, Laurentino Alves; ROCHA, Marcelo Pereira da. Aspectos relacionados à ocorrência de Flare-Up em Endodontia: Revisão da Literatura . Id on Line Rev.Mult. Psic., 2019, vol.13, n.45, p. 889899. ISSN: 1981-1179.

Recebido: 09/05/2019

Aceito 11/05/2019 